Table 1. Indipendent predictive variables in multivariate regression analysis and the resulting weighted prediction model *

\begin{tabular}{lccccc}
\hline Variable & $\boldsymbol{\beta}$ & HR & $95 \% \mathbf{C l}$ & $P$ & Weight \\
\hline Anti-Scl70 & 2.9553 & 19.21 & $4.87-75.76$ & $<0.001$ & 3.25 \\
Cs $\leq 5 / \mathrm{mm}$ & 1.9093 & 6.75 & $2.07-22.00$ & 0.001 & 2 \\
ANA $\geq 1: 320$ & 1.7402 & 5.70 & $1.42-22.85$ & 0.01 & 2 \\
ACA & 1.6740 & 5.33 & $1.51-1.90$ & 0.01 & 1.75 \\
GC $>5$ & 1.0049 & 2.73 & $1.44-5.17$ & 0.002 & 1 \\
\hline
\end{tabular}

${ }^{\star} \beta$ : regression coefficients; HR: hazard ratio; $95 \%$ Cl: 95\% confidence interval; Cs: Capillaries; ANA: anti-nuclear antibodies; ACA: anti-centromere antibodies; GC: giant capillaries

Conclusion: Assessing the mean number of capillaries/mm and the total number of giant capillaries instead of avascular areas at videocapillaroscopy, resulted in improving the sensitivity and specificity of the score recently developed to predict the evolution of UCTD-risk-SSc into definite SSc.

References:

[1] Valentini G. Autoimmun Rev 2015;

[2] Valentini G. et al. Arthritis Care Res (Hoboken). 2014;

[3] Riccardi A. et al. Autoimmun Rev. 2019;

[4] Sambataro et al. Arthritis Research \& Therapy 2014, 16:462.

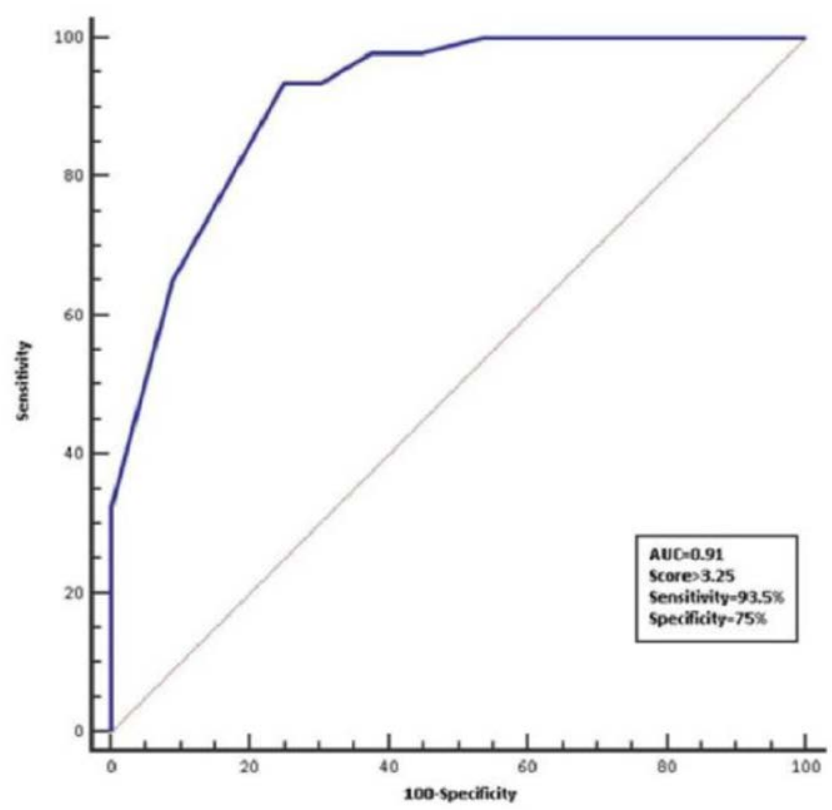

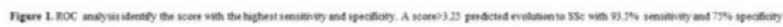

Disclosure of Interests: Antonella Riccardi: None declared, Antonella Marcoccia: None declared, SERENA FASANO: None declared, Tiziana Guastafierro: None declared, Rosaria Irace: None declared, Valentina Messiniti: None declared, Francesco Bondanini: None declared, Alessandro Sanduzzi: None declared, Marialuisa Bocchino: None declared, Aldo Ciani: None declared, Michele D'Alto: None declared, Paola Argiento: None declared, Giovanni Maria De Matteis: None declared, Alberto Spanò: None declared, Gabriele Valentini Grant/research support from: BMS, MSD, NOVARTIS, LILLY, PFIZER, ABBVIE, CELGENE

DOI: 10.1136/annrheumdis-2020-eular.3152

\section{SAT0341 PREDICTORS TO PROGRESSION TO SYSTEMIC SCLEROSIS IN A GROUP OF SECONDARY RAYNAUD PHENOMENON OBSERVED IN A LARGE SINGLE BRAZILIAN COHORT}

V. Siqueira ${ }^{1}$, M. Helbingen ${ }^{1}$, A. P. Luppino-Assad ${ }^{1}$, H. Carriço Da Silva ${ }^{1}$, D. Andrade ${ }^{1}$, A. C. Medeiros-Ribeiro ${ }^{1}$, P. D. Sampaio-Barros ${ }^{1,2}{ }^{1}$ University of Sao Paulo, Division of Rheumatology, São Paulo, Brazil; ${ }^{2}$ University of Sao Paulo, São Paulo, Brazil
Background: The 2013 ACR/EULAR classification criteria for systemic sclerosis (SSc) allowed the inclusion of a subset of patients without skin involvement, emphasizing the need of early diagnosis of SSc.

Objectives: In this setting, the objective of this study was to classify, according to ACR/EULAR SSc criteria, patients with Raynaud phenomenon (RP) and a least one SSc manifestation, except for skin involvement, and analyze predictors for development of SSc.

Methods: This is a cross-sectional single-center analysis of consecutive patients presenting RP and a characteristic SSc manifestation (SSc autoantibody, SSc visceral involvement or SD pattern at nailfold capillaroscopy - NFC), without skin involvement, who attended a scleroderma outpatient clinic between 2010 and 2019. Patients were classified as SSc (according to ACR/EULAR SSc criteria) or undifferetiated connective tissue disease (UCTD) in their last medical visit and compared. Additionally, a longitudinal retrospective analysis of both groups was performed to find predictors for development of SSc. Data were obtained from an electronic register database. Statistical significance was se up as $\mathrm{p}<0.05$.

Results: Among 217 patients, $153(70.5 \%)$ were classified as SSc, at the last medical visit, including $65(30 \%)$ after the first investigation. During a comparable median follow-up $[4.9 \pm 3.8$ vs. $4.6 \pm 3.0$ years; $p=0.90]$, patients with SSc presented more frequently puffy fingers (PF:73\% vs. $6.3 \% ; \mathrm{p}<0.01$ ), pitting scars ( $87.4 \%$ vs. $4.7 \%$; $<<0.01)$, digital ulcers $(25.2 \%$ vs. $1.6 \% ; p<0.01)$, telangiectasias (52.3\% vs. $7.8 \% ; p<0.01$ ), interstitial lung disease (ILD:66.7\% vs. $34.4 \% ; p<0.01$ ), pulmonary hypertension ( $\mathrm{PH}: 18.3 \%$ vs. $7.9 \% ; \mathrm{p}=0.049)$ and esophageal dismotility (ED: $58.9 \%$ vs. $43.8 \%: p=0.02$ ) than secondary RP patients without SSc Moreover, SSc patients had more SD pattern at NFC (83.4\% vs. 57.8\%;p<0.01), anti-Scl70 antibody (16.6\% vs. 3.1\%;p<0.01), and nucleolar ANA pattern $(22.5 \%$ vs. $10.9 \% ; \mathrm{p}=0.048)$. Furthermore, $\mathrm{SSc}$ patients were more frequently treated with immunosuppressive therapy $(80.1 \%$ vs. $37.5 \% ; p<0.01)$ and presented an increased death rate $(9.9 \%$ vs. $1.6 \% ; p<0.01$ and Figure 1$)$. For the analysis of the predictors to SSc, patients who had already fulfilled SSc criteria at baseline were excluded and 152 patients were analysed: 88 progressed to SSc and 64 remained as UCTD. After multivariate analyses, puffy fingers (OR=4.48; $95 \% \mathrm{Cl} 1.17-17.17 ; \mathrm{p}=0.029)$ and a higher SSc score, both at baseline, (OR: 1.97; $95 \% \mathrm{Cl} 1.33-2.92 ; \mathrm{p}<0.001)$ were predictors to SSc, while esophageal symptoms $(\mathrm{OR}=0.18 ; 95 \% \mathrm{Cl} 0.05-0.67 ; \mathrm{p}=0.01)$ and absence of clinical symptoms (OR=0.08; $95 \% \mathrm{Cl} 0.01-0.40 ; \mathrm{p}=0.002$ ) were negative predictive factors. When analyzing the combination of SSc features, RP + PF + positive ANA (OR=15.5 $95 \% \mathrm{Cl} 4.5-53.1 ; \mathrm{p}<0.001), \mathrm{RP}+\mathrm{PF}+$ positive ANA + SD-NFC $(\mathrm{OR}=12.3 ; 95 \% \mathrm{Cl}$ 2.8-54.2; $<<0.001), \mathrm{RP}+\mathrm{PF}+\mathrm{SD}-\mathrm{NFC}(\mathrm{OR}=12 ; 95 \% \mathrm{Cl} 2.79-54.1 ; \mathrm{p}<0.01)$, and $\mathrm{RP}+$ lung disease (ILD/HAP) + SD-NFC (OR=3.0; 95\%Cl 1.1-10.5; $<<0.04)$ were associated with classification as SSc, while combinations without non-RP clinical symptoms, as RP + SD-NFC (OR=0.027; 95\%Cl 0.003-0.210; $<<0.001)$ and RP + anticentromere + SD-NFC $(\mathrm{OR}=0.050 ; 95 \% \mathrm{Cl} 0.007-0.440 ; \mathrm{p}<0.001)$ were not associated with SSc.

Conclusion: Among patients with RP and SSc features, but without skin involvement, those with SSc had a more severe disease with worse prognosis than UCTD with RP. Puffy fingers and a higher baseline SSc score, especially in combinations including PF or lung disease were predictors of SSc, reinforcing the strength of the ACR/EULAR classification criteria and the presence of specific non-RP symptoms.

Disclosure of Interests: Valdirene Siqueira: None declared, Mariely Helbingen: None declared, Ana Paula Luppino-Assad: None declared, Henrique Carriço da Silva: None declared, Danieli Andrade: None declared, Ana Cristina Medeiros-Ribeiro: None declared, Percival D. Sampaio-Barros Consultant of: Abbvie, Boehringer Ingelheim, Lilly, Novartis, Speakers bureau: Abbvie, Janssen, Lilly, Novartis DOI: 10.1136/annrheumdis-2020-eular.4220

\section{SAT0342 \\ INNATE LYMPHOID CELLS ARE PREDICTORS OF DISEASE PROGRESSION IN SYSTEMIC SCLEROSIS: A 3-YEARS FOLLOW-UP STUDY}

A. Soare ${ }^{1}$, S. Weber ${ }^{1}$, G. Schett ${ }^{1}$, J. Distler ${ }^{1}$, A. Ramming ${ }^{1} .{ }^{1}$ Department of Internal Medicine 3, Rheumatology \& Immunology, 1 Friedrich-Alexander University (FAU) Erlangen-Nürnberg and Universitätsklinikum Erlangen, Erlangen, Germany

Background: Activation of the immune system is a characteristic feature of SSc. Numerous studies have suggested that type 2 are key drivers of progressive fibrosis. Recently, innate lymphoid cells (ILC) are emerging as an important cellular source of type 2 cytokines triggering fibrotic tissue remodeling independently of the adaptive immune system. Increased levels of ILC2 were found in patients with SSc. However, the contributive role of ILC2 in pathogenesis of SSc is not completely understood 
Objectives: We aim in our 3 years observational study to evaluate the predictive role of ILC2 in SSc patients.

Methods: We conducted an observational retrospective study on 52 patients with SSc fulfilling the 2013 ACR/EULAR classification criteria. Yearly clinical, laboratory and investigational data according to EUSTAR recommendations were collected. Blood samples collected between 15.09.2014 and 15.01.2015 were analyzed by flow cytometry and ILC2 counts were measured. The predictive value of ILC2 during a 3-year follow-up was analyzed using SPSS 21.0.

Results: 52 patients were included in the study, $78 \%$ female, $63 \%$ limited cutaneous SSc with a mean follow-up time of $2.85 \pm 1.28$ years. At baseline we have shown that circulating ILC2s are significantly increased compared to gender and age-matched healthy controls. Increased numbers of ILC2s significantly correlated with worsening of mRSS calculated by five point increase in $\mathrm{mRSS}$ or $25 \%$ increase from baseline $(p<0.001 ; 95 \% \mathrm{Cl} 1.39-3.26$ ). ILC2 counts also correlated with $5 \%$ decrease of diffusion capacity of carbon monoxide (DLCO) during the follow-up time ( $p<0.0001 ; 95 \% \mathrm{Cl} 1.83-3.49$ ). Worsening of forced vital capacity (FVC) assessed as $5 \%$ decrease over 2 years was also significantly correlated with an increased number of ILC2s $(p<0.0001 ; 95 \% \mathrm{Cl} 1.27-3.04)$. In contrast, we did not find any correlation regarding increase in pulmonary arterial pressure assessed by echocardiography. Although new appearance of digital ulcers could not be predicted by ILC2 counts, increased numbers of ILC2s were correlated with digital ulcers at follow-up.

Conclusion: Here, we provide first evidence for a role of ILC2s as potential prognostic marker of disease progression in SSc.

Disclosure of Interests: Alina Soare: None declared, Stefanie Weber: None declared, Georg Schett Speakers bureau: AbbVie, BMS, Celgene, Janssen, Eli Lilly, Novartis, Roche and UCB, Jörg Distler Grant/research support from: Boehringer Ingelheim, Consultant of: Boehringer Ingelheim, Paid instructor for: Boehringer Ingelheim, Speakers bureau: Boehringer Ingelheim, Andreas Ramming Grant/research support from: Pfizer, Novartis, Consultant of: Boehringer Ingelheim, Novartis, Gilead, Pfizer, Speakers bureau: Boehringer Ingelheim, Roche, Janssen

DOI: 10.1136/annrheumdis-2020-eular.6322

\section{SAT0343 NUTRITIONAL STATUS OF SYSTEMIC SCLEROSIS PATIENTS: A PILOT STUDY}

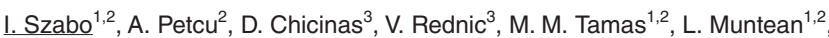
I. Filipescu ${ }^{1,2}$, S. P. Simon ${ }^{1,2}$, C. Pamfil ${ }^{2}$, S. Rednic ${ }^{1,2} .{ }^{1}$ County Emergency Clinical Hospital Cluj-Napoca, Rheumatology, Cluj-Napoca, Romania; ${ }^{2}$ University of Medicine an Pharmacy "Iuliu Hatieganu"Cluj-Napoca, ClujNapoca, Romania; ${ }^{3}$ Regional Institute of Gastroenterology and Hepatology "Octavian Fodor"Cluj-Napoca, Cluj-Napoca, Romania

Background: Gastrointestinal involvement (GI) in systemic sclerosis (SSc) is one of the major disease burdens. Its consequences on the nutritional status of SSc patients and their quality of life is poorly evaluated during routine check-ups. Since malnutrition is an important cause of morbidity and mortality, addressing this issue seems necessary.

Objectives: The aim of this study was to evaluate the risk of malnutrition in SSc patients and to identify potential associations between the risk of malnutrition and clinical features or laboratory parameters.

Methods: All patients aged $>18$ years old with a definite diagnosis of SSc according to the 2013 ACR/EULAR classification criteria from the EUSTAR Center 16 and ERN ReCONNET cohort of the County Emergency Clinical Hospital Cluj-Napoca were included in the study. Patients with localized scleroderma, scleroderma sine scleroderma, overlap syndromes and mixed connective tissue disease were excluded. Clinical and laboratory data was collected from the EUSTAR database and medical charts. A telephone survey was conducted and patients were interviewed using the Malnutrition Universal Screening Tool (MUST) questionnaire.

Results: 75 patients were eligible for the study. Female to male ratio was 10:1 with an almost equal distribution among limited (57\%) and diffuse (43\%) SSc subtypes. The most prevalent autoantibodies were anti-TOPO-I and anti-centromere. Gl symptoms were reported in $48.6 \%$ patients out of which $86 \%$ SSc patients underwent further evaluation by upper GI endoscopy. Abnormal endoscopic findings, such as esophagitis, Barret esophagus and gastritis were identified in $80 \%$ patients. Most patients had a low risk of malnutrition (93\%) with only a minority carrying a medium $(6 \%)$ or high $(1 \%)$ risk. No significant association was demonstrated between MUST score and the extend of cutaneous involvement (limited SSc versus diffuse SSc; $p=0.39$ ), presence of $\mathrm{Gl}$ symptoms $(p=0.35)$, presence of abnormal endoscopic findings $(p=0.45)$ or presence of anemia $(p=0.83)$
Conclusion: The majority of SSc patients from this cohort exhibited a low risk of malnutrition. These results are contradictory to previous literature reports. A possible explanation is that the MUST score is a dynamic screening too and therefore interviewing patients with a stable disease (outpatient care) versus patients with active disease (inpatient care) might lead to different results Another limitation of this study is the small number of patients included. This is a pilot study. We aim to further extend the study population to the other EUSTAR cohorts and to prospectively evaluate these patients in an inpatient care setting.

\section{References:}

[1] Dupont $\mathrm{R}$ et al. Impact of micronutrient deficiency \& malnutrition in systemic sclerosis: Cohort study and literature review. Autoimmun Rev. 2018 Nov;17(11):1081-1089;

[2] Caimmi C et al. Malnutrition and sarcopenia in a large cohort of patients with systemic sclerosis. Clin Rheumatol. 2018 Apr;37(4):987-997;

[3] Türk i et al. Malnutrition, associated clinical factors, and depression in systemic sclerosis: a cross-sectional study. Clin Rheumatol. 2020 Jan;39(1):57-67.

Disclosure of Interests: None declared

DOI: 10.1136/annrheumdis-2020-eular.6459

SAT0344 LIMITED JOINT MOBILITY OF HAND IN SYSTEMIC
SCLEROSIS PATIENTS BY USING "PRAYER" AND
"TABLE TOP" SIGNS

S. Uslu ${ }^{1}$, S. Gulle ${ }^{2}$, A. Koken Avsar ${ }^{2}$, A. Karakas² ${ }^{2}$ S. B. Kocaer ${ }^{2}$, T. Yüce İnel ${ }^{2}$, Y. Erez ${ }^{2}$, G. Can ${ }^{2}$, I. Sari ${ }^{2}$, F. Onen ${ }^{2}$, M. Birlik ${ }^{2} .{ }^{1}$ Ömer Halisdemir University Bor Physical Medicine and Rehabilitation, Training and Research Hospital, Department of Rheumatology, Niğde, Turkey; ${ }^{2}$ Dokuz Eylul University Faculty of Medicine, Department of Rheumatology, izmir, Turkey

Background: Limited joint mobility (LJM) is a musculoskeletal disorder caused by flexion contractures of hand is a common complication in systemic sclerosis (SSc) patients. The distal parts of the upper limb (hands and fingers) is the most involved site in SSc.

Objectives: In this study, we aimed to evaluate LJM in SSc patients and to determine the relationship between the clinical features of the disease.

Methods: A total of 113 patients ( $>18$ years old) diagnosed with diffuse cutaneous systemic sclerosis (DcSSc) and limited cutaneous systemic sclerosis (LcSSc) and 104 healthy controls were included in this study. LJM was evaluated with "prayer sign" and "table top sign" tests. LJM staging was done by Rosenbloom classification method(1, 2). LJM (+) and LJM (-) patients were compared in terms of demographic findings (gender, age and duration of disease), laboratory results (ESR, CRP, ANA, anti-topoisomerase I and anti-centromere) and modified Rodnan Skin Score (mRSS) results.

Results: In our study, a total of 113 patients diagnosed with SSc and 104 healthy controls with similar age and gender distribution were included. While LJM $(+)$ was detected in $75(66.4 \%)($ LcSSc $=38$, DcSSc $=37)$ of the patients diagnosed with SSc, LJM (mild) (+) was detected in $3(2.8 \%)$ of the control group. One of these people had right 2nd DIF joint contracture due to osteoarthritis, and 1 patient was found to have simple contractures due to minor hand injury previously (Table 1). A statistically significant difference was observed in between LcSSc and DcSSc patients according to the presence of LJM ( $<<0.001$ ) (Table 2). There was a moderate positivity relationship between LJM and mRSS (LcSSc: $r=0.449$ ve $p<0.001$, DcSSc: $r=0.565$ ve $p<0.001$ ) (Figure 1).

Table 1. Comparison of demographic findings between SSc and Control group

\begin{tabular}{|c|c|c|c|}
\hline & SSc Group ( $n=113)$ & Control Group ( $n=104)$ & $p$ value \\
\hline Age, year & $57.02 \pm 11.58$ & $58.47 \pm 11.26$ & 0.061 \\
\hline Gender (F / M) & $98(86.7) / 15(13.3)$ & 65 (62.5) / 39 (37.5) & 0.064 \\
\hline CRP (mg/L) & $5.45 \pm 5.39$ & $2.14 \pm 1.12$ & $<0.001$ \\
\hline ESR (mm/hr) & $25.19 \pm 18.9$ & $14.46 \pm 10.09$ & 0.024 \\
\hline \multicolumn{4}{|l|}{ Smoking, n (\%) } \\
\hline Smoker & 89 (78.8) & $70(67.3)$ & 0.464 \\
\hline Non-Smoker & 24 (21.2) & 34 (32.7) & \\
\hline \multicolumn{4}{|l|}{ LJM (Absent / Present) } \\
\hline Present & $75(66.4)$ & $3(2.8)$ & $<0.001$ \\
\hline Absent & $38(33.6)$ & $101(97.2)$ & \\
\hline Rosenbloom classification & $\operatorname{LcSSc}(n=71)(\%)$ & DcSSc $(n=42)(\%)$ & Total $(n=113)(\%)$ \\
\hline Normal & 46.5 & 11.9 & 33.6 \\
\hline Mild & 22.5 & 14.3 & 19.5 \\
\hline Moderate & 23.9 & 33.3 & 27.4 \\
\hline Severe & 7.1 & 40.5 & 19.5 \\
\hline
\end{tabular}

